\title{
Equivalent cyclic polygon of a euclidean travelling salesman problem tour and modified formulation
}

\author{
A. Herraiz ${ }^{1}$ (D) M. Gutierrez ${ }^{1}$ (D) $\cdot$ M. Ortega-Mier ${ }^{1}$ (D)
}

Accepted: 2 October 2021 / Published online: 2 December 2021

(C) The Author(s) 2021

\begin{abstract}
We define a geometric transformation of Euclidean Travelling Salesman Problem (TSP) tours that leads to a new formulation of the TSP. For every Euclidean TSP ncity tour, it is possible to construct an inscribed n-polygon (Equivalent Cyclic Polygon, ECP) such that the lengths of the edges are equal to the corresponding TSP tour links and follow the same sequence order. The analysis of the ECP elicits the possibility of defining a new objective function in terms of angles instead of distances. This modification opens the way to identify characterizing geometric parameters of the TSP as well as to explore new heuristics based on the inclusion of additional constraints. The experimentation with a set of cases shows promising results compared to the traditional compact formulations. The behavior of the ECP-based TSP formulations is better when the nodes of the TSP are randomly or evenly distributed.
\end{abstract}

Keywords Traveling salesman problem · Euclidean TSP · Equivalent cyclic polygon - TSP heuristic

\section{Introduction}

The Traveling Salesman Problem (TSP) is probably the most studied problem in combinatorial optimization. Biggs, Lloyd and Wilson (1976) trace back the academic attention received by the TSP to the nineteenth century. Since then, the problem has

$凶 \quad$ A. Herraiz

alvaro.herraiz.solla@alumnos.upm.es

M. Gutierrez

miguel.gutierrez@upm.es

M. Ortega-Mier

miguel.ortega.mier@upm.es

1 Ingeniería de Organización, Administración de Empresas y Estadística, Escuela Técnica Superior de Ingenieros Industriales, Universidad Politécnica de Madrid, José Gutiérrez Abascal 2, 28006 Madrid, Spain 
been extensively studied and there is a vast literature dealing with solving algorithms (Laporte 1992; Reinelt 1994; Raman and Gill 2017) and applications (Lenstra and Kan 1975; Junger et al. 1995). There have also been several formulations for the problem (Langevin et al. 1990; Orman and Williams 2007; Öncan et al. 2009).

The classic definition of the TSP can be stated as follows: Find the shortest route (tour) for a salesman starting from a given city, visiting each of a specified group of cities, and then returning to the original point of departure (Dantzig et al. 1954). The conceptualization of the problem can be generalized to minimize the duration or the total cost of the tour.

From the Graph Theory approach, the TSP can be stated as the problem of finding the least cost Hamiltonian circuit of a given complete graph $K_{\mathrm{N}} \equiv G(N, A)$, where $N$ $=\{1, \ldots, n\}$ is the set of nodes, and $A=\{(i, j): i, j \in N, i \neq j\}$ the set of arcs (Miller et al. 1960). Usually, the cost of each arc is defined by the cost (or distance) matrix $C$ $=\left\{c_{\mathrm{ij}}\right\}$. Depending on the relationship between $c_{\mathrm{ij}}$ and $c_{\mathrm{ji}}$, the problem is defined as Asymmetric TSP $\left(C \neq C^{T}\right)$ or Symmetric TSP $\left(C=C^{T}\right)$.

Several formulations have been proposed to solve the ATSP (Langevin Padberg and Sung 1991; Orman and Williams 1990; 2007). Öncan et al. (2009) provide a thorough analysis of the ATSP formulations, including how they relate to each other. To introduce our work and define the base model formulation, we summarize the main formulations, their basics, and distinctive approaches, and refer to this study for an in-depth analysis.

The work of Dantzig et al. (1954) is considered pioneer in the field. The DFJ formulation uses binary variables to formulate and solve the TSP. The authors propose what is called a natural formulation, in the sense that it does not include any additional variable other than $x_{\mathrm{ij}}$ to signify if the arc from $i$ to $j$ is selected for the tour or not. One essential aspect of TSP formulations is how to ensure that the solution is a single tour and not a set of independent subtours. To this purpose, in the DFJ formulation it is necessary to add a set of constraints that cannot be considered before starting the calculations without a sub-tour identification algorithm. Furthermore, the set of constraints grows exponentially with the number of cities of the TSP, $\mathrm{O}\left(2^{\mathrm{n}}\right)$, and, in practice, it is solved with delayed column generation methods.

To overcome these limitations, an alternative to the natural formulation is given by the so-called compact formulations. The basis for this set of formulations is provided by Miller et al. (1960). These authors propose the addition of instrumental variables that determine the order in which the cities are visited (their formulation is even more generic for they allow to visit all the cities in several tours, i.e., Vehicle Routing Problem (VRP); as it is the usual in TSP literature, in the remainder of the paper we will consider their model for the particular case of setting to one the number of tours (Öncan et al. 2009)). Thus, the MTZ formulation allows setting the subtour elimination constraints before solving the problem. Among the family of compact formulations, this approach is called a compact node-ordering formulation. The number of subtour elimination constraints compared to the DFJ formulation, is significantly reduced, $\mathrm{O}\left(n^{2}\right)$, although they lead to a weak linear programming (LP) relaxation. Desrochers and Laporte (1991) further develop the MTZ formulation and improve the performance of the subtour elimination constraints. 
Some other relevant compact formulations have been proposed. Gavish and Graves (1978) develop two compact arc-ordering or flow-based formulations with the intention to propose a formulation more adaptable to other transportation scheduling problems. They still use the same number of integer variables as MTZ, but they change the constraints set to include flow variables. Fox et al. (1980) provide another set of time-dependent TSP formulations. They reduce the number of linear constraints from $\mathrm{O}\left(n^{2}\right)$ to $\mathrm{O}(n)$ after including a set of time periods. Gouveia and Pires (2001) propose a compact precedence-based formulation, called the disaggregated MTZ model. They include a new variable that represents if a node is visited in the path between two other nodes.

As aforementioned, when the cost (distance) from one city $i$ to another city $j$ is the same if the arc is reversed $\left(c_{\mathrm{ij} j}=c_{\mathrm{ji}}, \forall_{i, j}\right)$, the resulting particular case of ATSP is called a Symmetric TSP (STSP). It is the case of the Euclidean TSPs in which the nodes represent points, and the costs are the corresponding Euclidean distances (symmetric by nature). The formulations for the ATSP are directly applicable to the STSP if the costs $c_{\mathrm{ij}}$ and $c_{\mathrm{ji}}$ are considered as if they were different, i.e., with two arcs of the same cost linking each pair of nodes. Nevertheless, for the natural formulation it is possible to achieve a more efficient STSP formulation if the two arcs connecting each pair of nodes are substituted by one single arc (Padberg and Sung 1991). In this case, the set of arcs will be $E=\{\{i, j\}: i, j \in \mathrm{N}, i<j\}$. Each arc will have a cost (or distance) $c_{\{\mathrm{ij}\}}$ associated as well as a decision binary variable $x_{\{\mathrm{ij}\}}$ similar to the ATSP formulation. The set of subtour elimination constraints is reduced in the STSP formulation compared to the ATSP formulation, hence the performance improvement, yet it remains exponential with the number of cities, making it necessary to include a subtour identification algorithm. For the MTZ and other compact formulations, "symmetrization" by means of considering one single arc linking each pair of nodes is not possible (Padberg and Sung 1991).

In this paper, we explore a geometric transformation of the Euclidean TSP that might open new possibilities of solving the TSP. The extensive literature on the problem has led to very efficient solving procedures. Nevertheless, the problem is known to be NPComplete (Papadimitriou 1977), and therefore, for problems with a large number of cities, the computation time becomes critical (Applegate et al. 2006; Dubois-Lacoste et al. 2015). On the other hand, there are numerous optimization problems that are variants and extensions of the TSP (Reinelt 1994; Anbuudayasankar et al. 2014). New approaches to the TSP can then be of great interest to be explored since they might foster applications in different problem variants.

Since the Euclidean TSP can be directly analyzed from a Euclidean geometric perspective, with the nodes representing points in the map and the tours being polygons, the interest of approaching the problem based on a geometric analysis has drawn the attention of researchers. One of the main geometric characteristics of the TSP is the fact that the optimal solution is a non-intersecting polygon (Lawler et al. 1991). Besides the analysis of geometric properties of the optimal solution, many works focus on the proposal of geometric-based heuristic algorithms (Lawler et al. 1991; Reinelt 1994). Some of them, like the largest angle heuristic (Norback and Love 1977) among many others (Asani et al. 2020), are based on the convex hull, which is the minimum convex polygon that encompasses a set of nodes. As computational 
geometry gained practical importance (Edelsbrunner 1987), there have been proposals based on techniques and concepts from this field aimed at solving the TSP. In particular, some of the methods are based on the application of Voronoi diagrams and Delaunay triangulations (Edelsbrunner 2014; Reinelt 1994).

However, mathematical properties of the TSP and the optimal solution remain open to research. Despite the great attention given to the TSP and its numerous applications and extensions, to the best of our knowledge there has not been a previous attempt of a geometric transformation of the tour polygon, so the problem can be formulated in terms of angles instead of distances. The main contribution of this paper is the proposal of such a geometric transformation of Euclidean Travelling Salesman Problem tours that leads to a modified formulation and new heuristic approaches, as well as to the characterization of intrinsic geometric properties of the solution. Experimentation with a set of problems shows that the proposed geometric transformation might lead to performance improvement in certain problems. The proposed approach opens the way to explore new solving strategies for this abundantly studied and applied problem.

In Sect. 2, we recall the compact node-ordering formulation from Miller et al. (1960). In Sect. 3, the proposed geometric transformation with the definition of equivalent polygonal chains and the equivalent cyclic polygon for every TSP tour is presented, leading to a modified formulation of the problem and new heuristic procedures that are presented in Sect. 4. In Sect. 5, we present the results yielded by the experimentation using a set of cases taken from TSPLIB (Reinelt 1991), which shows the potential practical interest of the proposed transformation. Finally, we summarize the main conclusions in Sect. 6.

\section{TSP base model formulation}

As aforementioned, in our work we focus on the geometric transformation of the Euclidean TSP tours and, thus, we deal with the Symmetric TSP. Based on this transformation, we define a modification of the objective function and explore the interest of adding some constraints. With the purpose of isolating the effect of the transformation and the addition of constraints, we want to compare our proposal directly solving a linear programming model that does not involve subtour elimination algorithms. For that reason, we do not take the symmetric DFJ as the base model formulation for the comparison. Among the compact formulations, we select as the base model formulation (Model A) the classic MTZ adapted to the TSP, which is widely known and sets the basis for this group of formulations.

Let us use the classical TSP formulation on a graph $G=(N, E)$, such that $N$ is the set of nodes (cities) $N=\{1,2, \ldots, n\}$, and $E=\{(i, j) \mid i, j \in N, i \neq j\}$ is the set of links. ${ }^{1}$ We use a distance parameter $d_{i j}$ for each $(i, j) \in E$, as the distance between nodes $i$ and $j$. We define a binary variable $x_{i j}$, which equals 1 if the edge $\{i, j\}$ is part of the tour, and 0 otherwise.

\footnotetext{
1 For the sake of clarity, we will use the terms nodes and links to refer to the TSP graph elements, vertices and edges to refer to the equivalent polygon elements, and the term arc to refer to a circular arc.
} 
Indices:

$i, j \in N$ Nodes.

Parameters:

$d_{i j} \quad$ Distance from node $\mathrm{i}$ to node $\mathrm{j}$.

$n=|N|$ Total number of nodes.

Variables:

$x_{i j} \in\{0,1\}, \forall(i, j) \in E \quad 1$ if we go from node $\mathrm{i}$ to node $\mathrm{j}, 0$ otherwise.

$u_{i} \geq 0, \forall i \in N \quad$ Auxiliary variables that determine the relative order in which node $\mathrm{i}$ belonging to the solution is visited.

Model A:

$$
\min z=\sum_{i, j} d_{i j} \cdot x_{i j}
$$

such that

$$
\begin{gathered}
\sum_{i} x_{i j}=1 \quad \forall j \\
\sum_{j} x_{i j}=1 \quad \forall i \\
u_{i}-u_{j}+1 \leq(n-1)\left(1-x_{i j}\right) \quad \forall i, j=2, \ldots, n, i \neq j
\end{gathered}
$$

Objective function (1) minimizes the total length of the tour. Constraints (2) and (3) guarantee that each node is visited only once. The set of constraints (4) prevents the presence of subtours in the solution while determining the sequence of the tour through the values of the auxiliary variables: if a link from node $i$ to node $j$ is in the solution $\left(x_{\mathrm{ij}}=1\right)$, then $u_{\mathrm{j}}$ has to be greater than $u_{\mathrm{i}}$ (for $i, j>1$, i.e., taking $i=1$ as the starting city of the tour). Constraints (4) constitute the grounds of the compact formulation.

Throughout the document, we will use the same definitions of indices, parameters, and variables of this section, whereas the different variants will concern the objective function and the set of constraints (which we will refer to as models, with Model A being the base model formulation).

\section{Proposed tour geometric transformation and modified TSP formulation}

The core of our proposal is the definition of a geometric transformation of any given feasible TSP tour. It is based on the construction of what we name "Equivalent Inscribed Polygonal Chain" (EIPC) and "Equivalent Cyclic Polygon" (ECP), which are described in detail in Sects. 3.1 and 3.2 respectively. 


\subsection{Equivalent inscribed polygonal chain}

Given a Euclidean TSP tour of $n$ cities defined by the sequence order in which they are visited $\left\{p_{1}, p_{2}, \ldots, p_{n}\right\}$, we define a geometric transformation such that a sequence of vertices $\left\{v_{1}, v_{2}, \ldots, v_{n}, v_{1}^{\prime}\right\}$ is inscribed in a circle of a given radius such that each link $p_{k-1}-p_{k}(k=2, \ldots, n)$ of the TSP tour has an equivalent edge $\overline{v_{k-1} v_{k}}$ of the same length, with the returning link $p_{n}-p_{1}$ equivalent to the last edge $\overline{v_{n} v_{1}^{\prime}}$. We note that in general there will be two vertices $\left(v_{1}, v_{1}^{\prime}\right)$ corresponding to the same city $p_{1}$. This transformation defines an inscribed polygonal chain (polyline) (Levcopoulos et al. 2002). The radio of the circumscribing circle, or circumcircle, is denoted as $R_{c}$.

To construct the equivalent inscribed polygonal chain (EIPC), we start positioning $v_{1}$ in any point of the circumcircle of radius $R_{c}$. Then, we define another circle with center in $v_{1}$ and radius equal to the length of link $p_{1}-p_{2}$, and find the intersection with the circumcircle. Since there will be two intersection points, we select $v_{2}$ in the circumcircle as the first intersection clockwise from $v_{1}$. We proceed the same way following the sequence of the remaining cities, until finding $v_{1}$ as the clockwise circumcircle intersection of the circle centered in $v_{n}$ and radius the length $p_{n}-p_{1}$.

If we define a Cartesian coordinate system $(x, y)$, placing the center of the circumcircle in the origin and $v_{1}$ in the vertical axis $\left(0, R_{c}\right)$ (or other specific point $\left.\left(x_{1}, y_{1}\right) \mid x_{1}^{2}+y_{1}^{2}=R_{c}^{2}\right)$, the vertices $v_{k}$ of the inscribed polygonal chain will verify the following (there will be two possible intersections for each vertex as abovementioned):

$$
\left.\begin{array}{c}
x_{k}^{2}+y_{k}^{2}=R_{c}^{2} \\
\left(x_{k}-x_{k-1}\right)^{2}+\left(y_{k}-y_{k-1}\right)^{2}=d_{k-1, k}^{2}
\end{array}\right\} \forall k
$$

and

$$
\left.\begin{array}{c}
x_{1^{\prime}}^{2}+y_{1^{\prime}}^{2}=R_{c}^{2} \\
\left(x_{1^{\prime}}-x_{n}\right)^{2}+\left(y_{1^{\prime}}-y_{n}\right)^{2}=d_{n, 1^{\prime}}^{2}
\end{array}\right\}
$$

Figure 1 shows an example of an 8-city TSP tour defined by the sequence 1-2-8-37-4-5-6 (middle of the figure) and two EIPCs. Depending on the length of the radius, the summation of all the central angles defined by the chain edges will be higher than $2 \pi$ (left) or lower than $2 \pi$ (right). In the first case (left), non-consecutive edges of the chain will intersect (self-intersecting polygonal chain (Levcopoulos et al. 2002)).

\subsection{Equivalent cyclic polygon}

In the example of Fig. 1, if we progressively decrease the radius of the right chain or increase the radius of the left chain, by continuity (Macnab 1981), there will be a radius in which $v_{1}^{\prime}$ will converge to $v_{1}$. The polygonal chain will then close, resulting in what we call Equivalent Cyclic Polygon (ECP) of a TSP tour. In this section, we provide a formal definition of the ECP, a detailed algorithm for its construction as well as for the calculation of the radius of the circumcircle. 

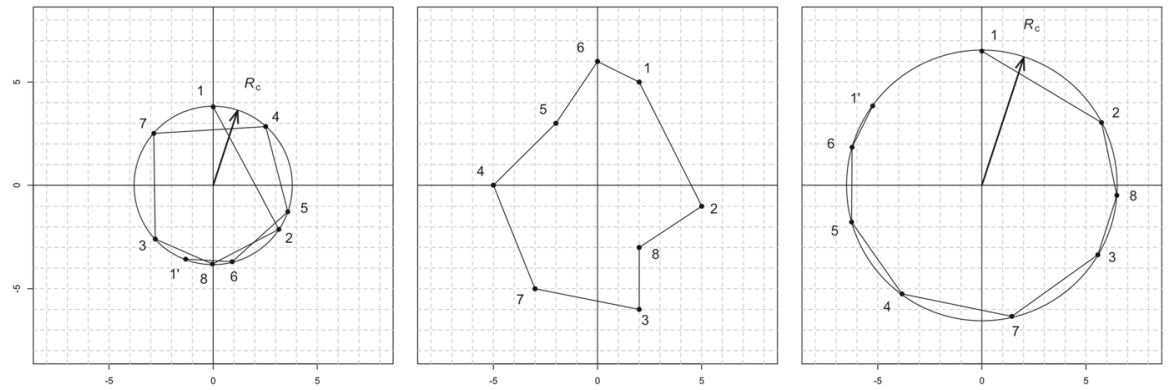

Fig. 1 Example of two possible geometric transformations of a TSP tour and the resulting equivalent inscribed polygonal chains (EIPC)

The definition of the ECP of a TSP tour is a particularization of a more general concept from geometry. Panina and Khimshiashvilib (2012) define a polygonal linkage as "a set of positive numbers $L=\left(l_{1}, l_{2}, \ldots, l_{\mathrm{n}}\right) \in \mathbb{R}_{+}^{n}$, which can be realized as the lengths of edges of a closed polygonal line. A $d$-dimensional configuration of a polygonal linkage is a set of points $p_{1}, p_{2}, \ldots, p_{\mathrm{n}}, p_{\mathrm{i}} \in \mathbb{R}^{d}$, satisfying the conditions $\left|p_{\mathrm{i}} p_{\mathrm{i}+1}\right|=l_{\mathrm{i}},\left|p_{\mathrm{n}} p_{1}\right|=l_{\mathrm{n}}$, where $|\cdot|$ denotes the Euclidean distance between two points. Physically, a polygonal linkage is interpreted as a set of cyclically joined rigid rods of length $l_{\mathrm{i}}$. At the locations of joints (hinges), free bending and, thereby, selfintersections and self-overlaps of edges are allowed."

Among the set of possible configurations of a polygonal linkage, called the moduli space, we focus on cyclic polygons, i.e., polygons in which all vertices are on a circle. Pinelis (2005), extending and complementing the work of Macnab (1981), proves the existence and isometric uniqueness of a convex cyclic polygon with edges that follow a given sequence of lengths (in which none is larger than the sum of the others). In the case of the proposed geometric transformation of a TSP tour, the proof of Pinelis (2005) assures the existence and uniqueness the ECP, since a tour defines a sequence of lengths. We define the ECP as follows.

Given a Euclidean TSP tour of $n$ cities defined by the sequence order in which they are visited $\left\{p_{1}, p_{2}, \ldots, p_{n}\right\}$, it is possible to construct an inscribed $n$-polygon with vertices in sequence $\left\{v_{1}, v_{2}, \ldots, v_{n}\right\}$ such that each link $p_{k-1}-p_{k}(k=2, \ldots, n)$ of the TSP tour has an equivalent polygon edge $\overline{v_{k-1} v_{k}}$ of the same length, with the returning link $p_{n}-p_{1}$ equivalent to the last edge $\overline{v_{n} v_{1}}$. The circumcircle of the ECP is called the mass circle, with radius $R_{m}$ called the mass radius.

Figure 2 represents the ECP of the example of Fig. 1 and illustrates the basic relationship between $R_{m}$, the length of each edge, and the central angle that it delimits. Let us consider the link from node $i$ to node $j$ belonging to a tour. Then the relation of Eq. (7) ( $i=1$ and $j=2$ in Fig. 2), is verified for $\alpha_{\mathrm{ij}} \leq \pi$.

$$
\frac{d_{i j}}{2}=R_{m} \sin \left(\frac{\alpha_{i j}}{2}\right), \alpha_{i j}=2 \cdot \arcsin \left(\frac{d_{i j}}{2 \cdot R_{m}}\right), \forall(i, j) \in E \mid x_{i j}=1
$$



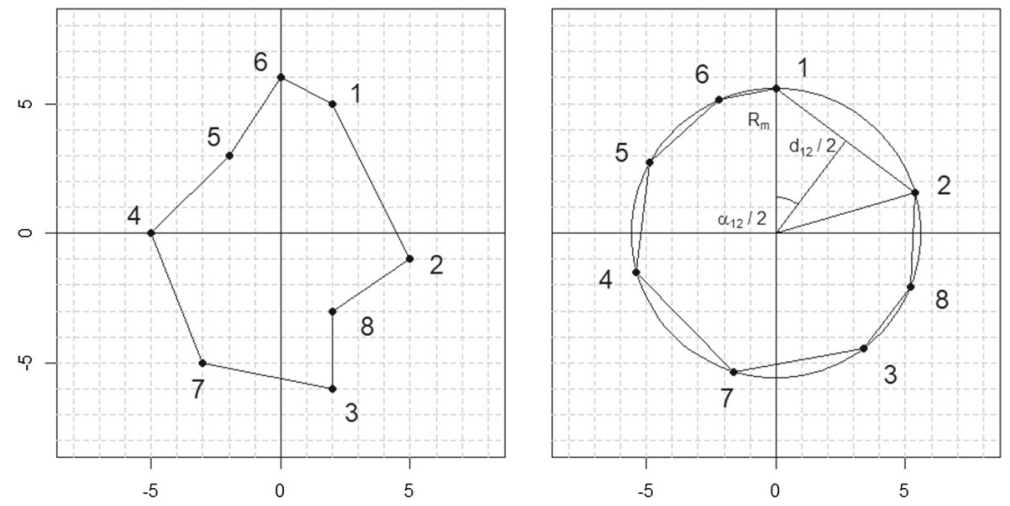

Fig. 2 Example of a TSP tour (left) and its ECP (right)

Summation of all the central angles of the ECP will equal $2 \pi$ (Eq. 7). Thus, given a TSP tour and the set of distances of the links $\left\{d_{\mathrm{ij}}, x_{\mathrm{ij}}=1\right\}$ such that all the central angles verify $\alpha_{i j} \leq \pi$ with $d_{\max }=\max _{i j}\left(d_{i j}\right), x_{i j}=1$, we can calculate $R_{m}$ by solving the transcendental Eq. (7) either with the aid of mathematical software or through an iterated procedure.

$$
\sum_{i, j} \arcsin \left(\frac{d_{i j}}{2 R_{m}}\right)=\pi, R_{m} \geq \frac{d_{\max }}{2}, \forall(i, j) \in E \mid x_{i j}=1
$$

To gain insights into the existence and uniqueness of the ECP, and in an equivalent approach to Pinelis (2005), we define the function $F_{1}$ in Eq. (8), such that Eq. (7) can be expressed as $F_{1}\left(R_{m}\right)=0$.

$$
F_{1}\left(R_{c}\right)=\sum_{i j} \arcsin \left(\frac{d_{i j}}{2 R_{c}}\right)-\pi, R_{c} \geq \frac{d_{\max }}{2}, \forall(i, j) \in E \mid x_{i j}=1
$$

$F_{1}$ is continuous, differentiable, and strictly decreasing in the interval $\left[d_{\max } / 2\right.$, $\infty)$. Besides, $F_{1}$ converges asymptotically to $-\pi$ as $R_{c}$ tends to infinity. Then, if $a=$ $F_{1}\left(d_{\max } / 2\right) \geq 0$, there will be a unique value of $R_{\mathrm{m}} \mid F_{1}\left(R_{\mathrm{m}}\right)=0$. Figure 3 shows the representation of $F_{1}\left(R_{c}\right)$ for the example of Fig. 2.

If the tour has the particularity of having a very large link, it might happen that Eq. (7) cannot be satisfied even for the minimum possible mass radius $R_{m}=d_{\max } / 2$. In this case, the EIPC does not close even for the minimum $R_{m}$, for which the corresponding edge with maximum length would be the diameter of the circumcircle. The central angle of the longest edge would equal $\pi$, whereas the sum of the rest of the central angles would be less than $\pi$. From Eq. (8) this case would then verify that $a<0$, implying $F_{1}<0$ in all its domain. Conceptually, the fact that $a<0$ means that, for the ECP to be closed, all of its vertices have to belong to a semicircle. This can be stated as the central angle of the longest link being larger than $\pi$. Figure 4 illustrates this 


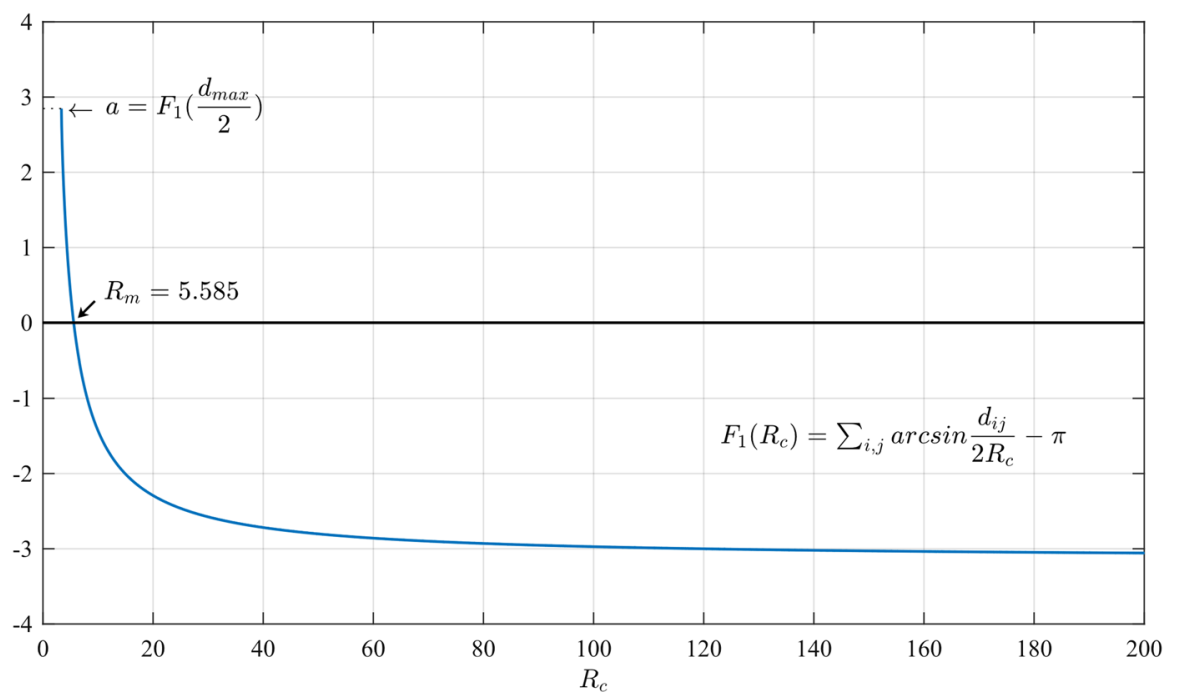

Fig. 3 Plot of function $F_{1}\left(R_{\mathrm{c}}\right)$ for the cities of Fig. 2 example
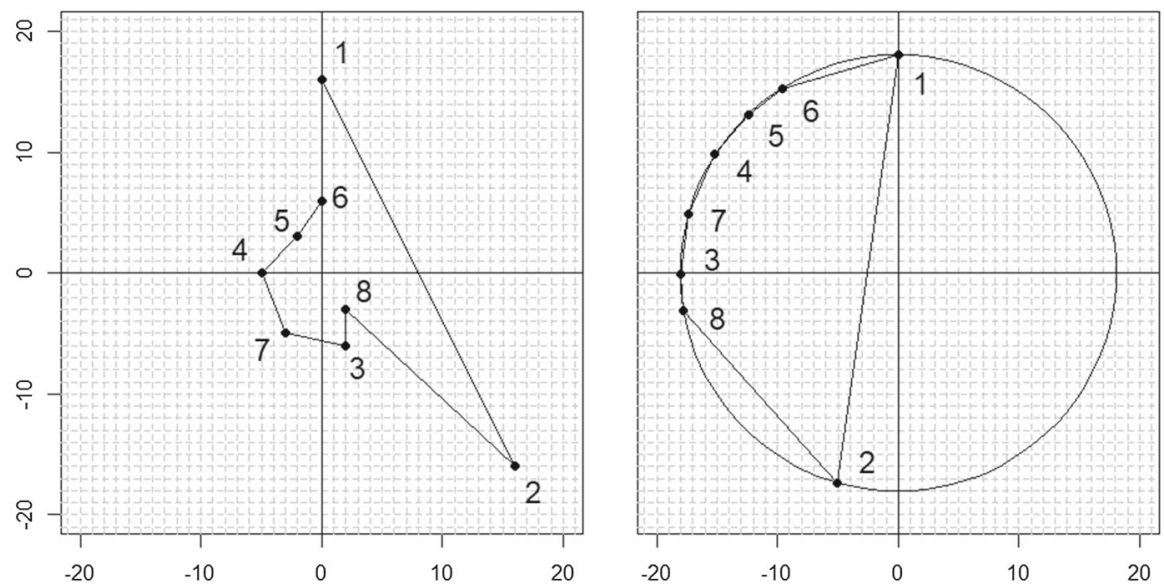

Fig. 4 Example of a TSP tour (left) and its ECP (right) for the case $a<0$

fact through a very simple example (obtained by enlarging link 1-2 in the example of Fig. 2).

For most of the TSP tours $a \geq 0$ and the construction of the ECP is identical to the procedure described in the former subsection for the construction of the EIPC. Yet, the possibility of $a<0$ requires introducing a modification as follows. For the longest link, the vertex must be determined by taking the counterclockwise intersection. Thus, the central angle will be $2 \pi-2 \cdot \arcsin \left(\frac{d \max }{2 R_{m}}\right)$. Subtracting the central angle from the summation and adding the counterclockwise angle (divided by 2) in Eq. (7) yields 
the condition that must verify $R_{\mathrm{m}}$ (Eq. 9).

$$
\begin{aligned}
& {\left[\sum_{i j} \arcsin \left(\frac{d_{i, j}}{2 R_{m}}\right)-\arcsin \left(\frac{d_{\max }}{2 R_{m}}\right)\right]+\left[\pi-\arcsin \left(\frac{d_{\text {max }}}{2 R_{m}}\right)\right]=\pi} \\
& \sum_{i, j} \arcsin \left(\frac{d_{i j}}{2 R_{m}}\right)-2 \cdot \arcsin \left(\frac{d_{\text {max }}}{2 R_{m}}\right)=0, R_{m} \geq \frac{d_{\max }}{2}, \forall(i, j) \in E \mid x_{i j}=1
\end{aligned}
$$

Analogously to the case $a \geq 0$, the function $F_{2}$ of Eq. (10) is defined, and Eq. (9) can be expressed as $F_{2}\left(R_{\mathrm{m}}\right)=0$. We note that for $R c=\left(d_{\max } / 2\right)$, the second term of $F_{2}$ equals $\pi\left(\arcsin \left(d_{\max } / 2 R_{\mathrm{c}}\right)=\arcsin (1)=\pi / 2\right)$ and hence $F_{1}$ and $F_{2}$ have the same expression. Therefore, $a=F_{1}\left(d_{\max } / 2\right)=F_{2}\left(d_{\max } / 2\right)$.

$$
F_{2}\left(R_{c}\right)=\sum_{i j} \arcsin \left(\frac{d_{i j}}{2 R_{c}}\right)-2 \cdot \arcsin \left(\frac{d_{\max }}{2 R_{c}}\right), R_{c} \geq \frac{d_{\max }}{2}, \forall(i, j) \in E \mid x_{i, j}=1
$$

$F_{2}$ is differentiable in the interval $\left[d_{\max }, \infty\right)$ and converges asymptotically to $0^{+}$as $R_{c}$ tends to infinity. A detailed analysis of the function, its first derivative and curvature, shows that $F_{2}^{\prime}$ tends to infinity as $R_{\mathrm{c}}$ tends to $d_{\text {max }} / 2\left(F_{2}\right.$ has vertical slope for $R_{\mathrm{c}}=$ $d_{\max } / 2$ ), and has a unique zero which corresponds to a $F_{2}$ maximum. If $a<0, F_{2}$ will have different signs in the extremes of the domain, asymptotic convergence to $0^{+}$, and there will be a unique value of $R_{\mathrm{m}} \mid F_{2}\left(R_{\mathrm{m}}\right)=0$ (see Pinelis (2005) for an equivalent thorough proof). Figure 5 shows the representation of $F_{2}\left(R_{c}\right)$ for the example of Fig. 4.

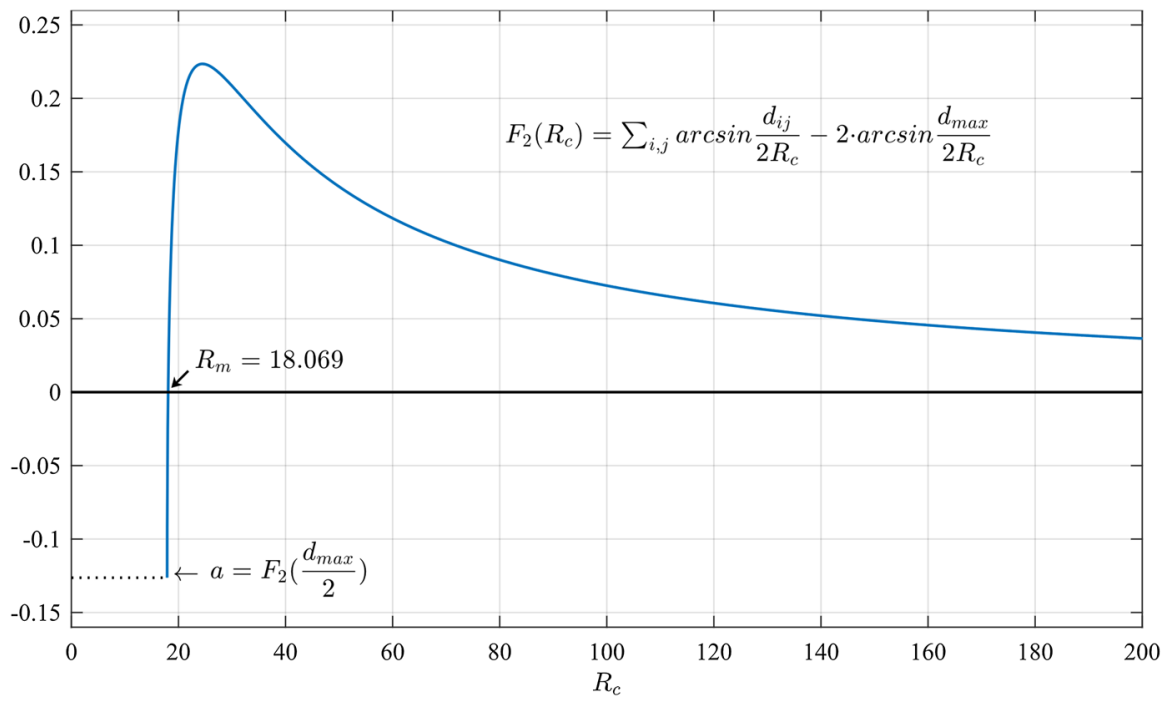

Fig. 5 Plot of function $F_{2}\left(R_{\mathrm{c}}\right)$ for the cities of Fig. 4 example in which a $<0$ 
We can then define the following procedure to construct the ECP of a TSP tour, based on the EIPC construction as described in Sect. 3.1:

Step 1 Define the sequence of cities such that the link $p_{1}-p_{2}$ is the longest.

Step 2 Compute $a=F_{1}\left(d_{\max } / 2\right)$.

Step 3 Find $R_{\mathrm{m}}$.

- If $a \geq 0$. Find $R_{\mathrm{m}} \mid F_{1}\left(R_{\mathrm{m}}\right)=0$.

- If $a<0$. Find $R_{\mathrm{m}} \mid F_{2}\left(R_{\mathrm{m}}\right)=0$.

Step 4 Construct the ECP.

- If $a \geq 0$. Proceed as described in the construction of the EIPC.

- If $a<0$. Proceed as described in the construction of the EIPC, but with the following modification: select $v_{2}$ in the circumcircle as the first intersection counterclockwise from $v_{1}$.

We can gain some insights on the ECP and the TSP with many nodes, analyzing the case in which the distances between the cities of the tour are of equal length $d$. The ECP in this case will be a regular polygon. Let us consider a parameter $\gamma>1$, that will represent the relation between the length of the mass circle and the length of the ECP of an $n$-city TSP as defined by Eq. (11). As the number of number of cities of the problem grows, the length of the tour will be closer to the length of the mass circle (i.e., $\gamma \rightarrow 1$ ).

$$
2 \pi R_{m}=\gamma \cdot n \cdot d \rightarrow \gamma=\frac{2 \pi R_{m}}{n \cdot d}
$$

The definition of the parameter $\gamma$ can be generalized, so it can be applicable to any TSP tour as the inverse relation of the length of the tour and the length of its mass circle. In general, for a TSP with many equally distributed nodes, $\gamma$ will be close to 1. A value of $\gamma$ significantly higher than 1 will indicate the presence of large links in the TSP tour. Thus, $\gamma$ can be used as one parameter that characterizes the topology of a TSP tour (Eq. 12) and, moreover, to characterize the TSP as follows. The optimal TSP tour allows us to construct its corresponding ECP*, with mass radius $R_{m} *$. Thus, if the parameter $\gamma$ is calculated for the optimal tour, it will then be a characterizing parameter of the TSP.

$$
\gamma^{*}=\frac{2 \pi R_{m}^{*}}{\sum_{i j} d_{i j} x_{i j}}, \forall(i, j) \in E \mid x_{i j}=1
$$

\section{ECP-based TSP formulation and heuristics}

The geometric transformation of the TSP tours leads to a modified formulation of the problem. As the circumradius of a tour EIPC tends to infinity, the length of each edge converges to the corresponding arc of the circumcircle. For a large enough circumradius, the longer the tour, the greater the total central angle in its EIPC. Therefore, the 
objective function of the TSP (Eq. 1) can be substituted by the sum of EIPC half central angles for a circumference of a large enough circumradius $\left(R_{c}^{L}\right)$, yielding an equivalent formulation (13). If we express the circumradius as a factor times the minimum possible radius to include any link of the TSP, it is verified that: $R_{c}^{L}=q \cdot d_{\max } / 2, q \gg 1$.

Instead of the standard approach of minimizing the sum of costs or distances (1), the objective function can be formulated as a minimization of the sum of the half central angles of a transformed tour (13). Taking the same indices, parameters, variables, and constraints (Eqs. 2-4) of the formulation of Miller et al. (1960) included in Sect. 2, we can define the new ECP-based TSP (ECPTSP) formulation as follows.

$$
\min z=\sum_{i, j} x_{i j} \arcsin \left(\frac{d_{i j}}{2 R_{c}^{L}}\right)
$$

such that

$$
\begin{gathered}
\sum_{i} x_{i j}=1 \quad \forall j \quad \text { (2) } \\
\sum_{j} x_{i j}=1 \quad \forall i \quad \text { (3) } \\
u_{i}-u_{j}+1 \leq(n-1)\left(1-x_{i j}\right) \quad \forall i, j=2, \ldots, n, i \neq j
\end{gathered}
$$

The proposed modified formulation opens the way to define a new family of heuristics for the TSP, through the specification of different values for the circumradius and the addition of EPC geometric constraints. In the following subsections, we propose three heuristics based on the geometric transformation that are modified formulations of the MTZ base model formulation (Sect. 2).

\subsection{Model B-heuristic mass radius (ECPTSP_hmR)}

The first heuristic strategy consists in reducing the radius of the ECPTSP formulation objective function (Eq. 13), so the formulation helps to guide the algorithm by avoiding large links (both explicitly and implicitly) while aligning with the objective of the minimum distance. This is done by solving the TSP with a fast heuristic and taking the mass radius of the ECP of the heuristic solution $\left(R_{m}^{H}\right)$ as the radius for the objective function. For the experimentation of Sect. 5, we use the nearest neighbor heuristic - starting from an arbitrary city, iteratively select the city that is closest to the last city included in the tour-, which is simple and fast, although it is known to yield low quality solutions with large returning links (Rosenkrantz et al. 1977). In fact, for all cases of the experimentation (Sect. 5) it is verified that $R_{m}^{H}=q \cdot d_{\max } / 2, q>1$.

To complete the modified formulation, it is also necessary to add the set of constraints (15) that express the impossibility for an edge to surpass the diameter of the mass circle, assuring the range of validity of the arcsine functions in the objective function. 
Model B:

$$
\min z=\sum_{i, j} x_{i j} \arcsin \left(\frac{d_{i j}}{2 R_{m}^{H}}\right)
$$

Such that.

$$
\begin{gathered}
\sum_{i} x_{i j}=1 \quad \forall j \quad \text { (2) } \\
\sum_{j} x_{i j}=1 \quad \forall i \quad \text { (3) } \\
u_{i}-u_{j}+1 \leq(n-1)\left(1-x_{i j}\right) \quad \forall i, j=2, \ldots, n, i \neq j \\
d_{i j} x_{i j} \leq 2 R_{m}^{H} \forall i, j
\end{gathered}
$$

We underline the elimination of links that derives from Eq. (15) and its practical application to reduce the computation time for large problems. On the contrary, if the topology of the problem is such that the optimal solution includes large links, ECPTSP_hmR formulation might not provide quality solutions, particularly if a better heuristic is employed. In general, as we develop in the remainder of the paper, there is a trade-off between properly accelerating the search and leaving aside good solutions.

\subsection{Model C—summation of arcs constraint (ECPTSP_summarcs)}

The ECPTSP formulation also opens the possibility of adding a constraint that might improve the calculations for complex problems. The idea behind the additional cut lies in the fact that, in general, for problems of large size, tours in which the total central angle of the circumcircle of the heuristic mass radius exceeds $2 \pi$ are likely to be low quality solutions. The ECPTSP_summarcs formulation (Model C) results from the addition of constraint (16) to the ECPTSP_hmR formulation.

Model C:

$$
\min z=\sum_{i, j} x_{i j} \arcsin \left(\frac{d_{i j}}{2 R_{m}^{H}}\right)
$$

such that

$$
\begin{gathered}
\sum_{i} x_{i j}=1 \quad \forall j \quad \text { (2) } \\
\sum_{j} x_{i j}=1 \quad \forall i \quad \text { (3) } \\
u_{i}-u_{j}+1 \leq(n-1)\left(1-x_{i j}\right) \quad \forall i, j=2, \ldots, n, i \neq j
\end{gathered}
$$




$$
\begin{gathered}
d_{i j} x_{i j} \leq 2 R_{m}^{H} \quad \forall i, j \\
\sum_{i, j} x_{i j} \arcsin \left(\frac{d_{i j}}{2 R_{m}^{H}}\right) \leq \pi
\end{gathered}
$$

\subsection{Model D—Small Circumradius (ECPTSP_sCR)}

The next step in the trade-off between reduction of the search space and not eliminating good solutions is explored by drastically reducing the radius of the ECPTSP objective function. Instead of using a large $R_{\mathrm{c}}{ }^{L}$ in the objective function (13), we can use what we call a small circumradius $R_{\mathrm{c}}{ }^{S}$ that is smaller than half the maximum distance $R_{c}^{S}=q \cdot d_{\max } / 2, q<1$. The formulation is completed by adding a set of constraints equivalent to (15).

Model D:

$$
\min z=\sum_{i, j} x_{i j} \arcsin \left(\frac{d_{i j}}{2 R_{c}^{S}}\right)
$$

such that

$$
\begin{gathered}
\sum_{i} x_{i j}=1 \quad \forall j \quad \text { (2) } \\
\sum_{j} x_{i j}=1 \quad \forall i \quad \text { (3) } \\
u_{i}-u_{j}+1 \leq(n-1)\left(1-x_{i j}\right) \quad \forall i, j=2, \ldots, n, i \neq j \\
d_{i j} x_{i j} \leq 2 R_{c}^{S} \quad \forall i, j
\end{gathered}
$$

To have the possibility of achieving the optimal value, $R_{c}{ }^{S}$ can be reduced as long as no arc of the optimal solution is unable to satisfy constraints (18). Lowering $R_{C}{ }^{S}$ might also lead to avoiding the optimum yet yielding a feasible solution. The challenging issue is how to set the value of $R_{\mathrm{c}}{ }^{S}$ low enough to significantly reduce the number of edges while not making it hard to find a feasible quality solution. It is immediate to determine that it should never be smaller than half the minimum distance from a node to the others, since, if it is not, any link including that node will not satisfy Eq. (18) (it will not fit into the circumcircle). Finding a suitable value for $R_{\mathrm{c}}{ }^{S}$ is an issue that arises from the proposed ECPTSP-scR formulation and is subject to research. Noteworthy, due to the reduced value of $R_{C}{ }^{S}$, the objective function value for most tours will likely exceed $2 \pi$ (as in Fig. 1 left). For the experimentation of Sect. 5, we take $R_{\mathrm{c}}{ }^{S}$ as the longest among the minimum distances from each node to the others $R_{c}^{S}=\max _{j}\left(\min _{i} d_{i j}\right)$, which, for all the cases selected for experimentation verify $R_{c}^{S}=q \cdot d_{\max } / 2, q<1$. 


\section{Experimentation}

Aiming to assess the practical interest of the proposed geometric transformation as well as the different heuristic formulations presented in the former section, we compare the following 4 formulations of the TSP:

- Model A. Classical formulation of Miller et al. (1960) (TSP base model formulation as defined in Sect. 2).

- Model B. ECPTSP_hmR formulation, computing $R_{m}{ }^{H}$ with the nearest neighbor heuristic tour (Rosenkrantz et al. 1977).

- Model C. ECPTSP_summarcs formulation with the same $R_{m}{ }^{H}$ of Model B.

- Model D. ECPTSP_scR formulation, with $R_{C}{ }^{S}$ calculated as the longest among the minimum distances from each node to the others.

\subsection{Experimental settings}

To perform the above comparison, from TSPLIB (Reinelt 1991) we select the 40 TSPs in the Euclidean plane and symmetric (Symmetric EUC_2D type) with a maximum of 400 nodes. Due to the nature of the proposed geometric transformation, it is necessary to compute float Euclidean distances when solving the TSPs. We note that this is not the standard stablished for the TSPLIB instances, in which distances are rounded to integer values. In order to have a comparison benchmark, for each TSPLIB instance, the floating Euclidean distance of the known optimal tour has been calculated, even though for some cases those tours are not optimal.

The experimentation has been performed using a computer with Intel(R) Core (TM) i3-8100 CPU @ 3.60 GHz. We formulate all models in Python 2.7, using the Pyomo package to code the formulation (Hart et al. 2017), the Gurobi 9.1 solver (Gurobi 2021a) to find a solution, and the R programming language to plot the nodes and results (R Core Team 2020). For all runs, a time limit of 10,000 s and a Gurobi optimality MIPGap (Relative MIP optimality gap, Gurobi 2021b) of $0.01 \%$ was set for each run. MIPGap provides a relative maximum distance to the optimal value. It is the MIP primal-dual relative gap, calculated as the absolute difference between the incumbent objective function value (primal upper bound) and the incumbent dual objective function value (lower bound) of a Mixed Integer Programming (MIP) problem, relative to the incumbent primal objective value (Gurobi 2021b).

\subsection{Results}

Table 1 shows the results divided in three groups. Table 1a includes the results for those instances in which all formulations consumed the time limit without achieving the MIPGap (time $=10,000 \mathrm{~s}$ for all runs). Table $1 \mathrm{~b}$ includes the results in which at least one formulation achieved the MIPGap without reaching the time limit and at least one consumed the time limit (therefore it specifies the computation time). Finally, Table 1c includes those cases in which all formulations reached the MIPGap of $0.01 \%$. 
Table 1 TSPLIB experimentation results

\begin{tabular}{|c|c|c|c|c|c|c|c|c|c|}
\hline \multirow[t]{2}{*}{ TSPLIB case } & \multicolumn{4}{|c|}{ Gap (\%): } & \multirow[t]{2}{*}{$\theta$} & \multirow{2}{*}{$\begin{array}{l}\text { z (length) } \\
\text { A }\end{array}$} & \multicolumn{3}{|c|}{ z (angle) } \\
\hline & A & B & $\mathrm{C}$ & $\mathrm{D}$ & & & B & $\mathrm{C}$ & $\mathrm{D}$ \\
\hline \multicolumn{10}{|c|}{ a. All runs consumed $10,000 \mathrm{~s}$} \\
\hline $\operatorname{ch} 130$ & 7.87 & 0.53 & 0.28 & 2.24 & 0.949 & $6,633.10$ & 2.52 & 2.51 & 43.53 \\
\hline $\operatorname{ch} 150$ & 3.80 & 1.60 & 0.31 & -0.31 & 1.133 & $6,810.24$ & 2.53 & 2.49 & 38.45 \\
\hline d198 & 22.00 & 10.06 & 17.38 & 21.52 & 0.440 & $20,413.72$ & 2.49 & 2.71 & 9.20 \\
\hline gil262 & 1.60 & 0.67 & 7.49 & -0.58 & 0.994 & $2,439.59$ & 2.29 & 2.46 & 62.76 \\
\hline kroA 150 & 1.65 & 1.63 & 5.36 & -0.41 & 1.019 & $27,080.24$ & 2.51 & 2.60 & 45.14 \\
\hline kroA200 & 14.65 & 20.55 & - & 0.33 & 0.984 & $34,479.83$ & 3.23 & - & 50.32 \\
\hline kroB100 & -0.79 & -0.79 & -0.79 & -0.79 & 1.027 & $22,139.07$ & 2.28 & 2.28 & 24.53 \\
\hline kroB 150 & 2.10 & -0.21 & -1.30 & -1.30 & 1.004 & $27,043.09$ & 2.49 & 2.47 & 37.42 \\
\hline kroB200 & 15.10 & 9.62 & 8.07 & -0.32 & 1.003 & $34,804.09$ & 2.75 & 2.71 & 59.98 \\
\hline kroC100 & -0.79 & 12.57 & -0.79 & -0.79 & 1.013 & $20,750.76$ & 2.81 & 2.41 & 29.99 \\
\hline kroD100 & 10.54 & -0.18 & -1.19 & -1.19 & 1.003 & $24,101.70$ & 2.44 & 2.41 & 27.56 \\
\hline $\operatorname{lin} 105$ & 0.00 & 7.48 & 9.90 & 4.21 & 0.772 & $14,382.99$ & 2.21 & 2.27 & 26.95 \\
\hline $\operatorname{lin} 318$ & 13.57 & 13.56 & - & 2.18 & 0.727 & $48,642.09$ & 2.78 & - & 76.45 \\
\hline $\operatorname{linhp} 318$ & 13.57 & 13.56 & - & 2.18 & 0.727 & $48,642.09$ & 2.78 & - & 76.45 \\
\hline pr107 & 8.64 & 15.53 & - & - & 0.554 & $56,210.72$ & 3.51 & - & infeas \\
\hline pr124 & 0.23 & 0.43 & -0.27 & - & 0.683 & $59,607.74$ & 2.54 & 2.53 & - \\
\hline pr136 & 7.54 & 12.18 & 7.28 & - & 1.255 & $104,570.26$ & 2.87 & 2.71 & infeas \\
\hline pr144 & 28.39 & 33.86 & - & - & 0.326 & $82,365.50$ & 4.41 & - & - \\
\hline pr152 & 24.96 & 30.32 & - & - & 0.457 & $98,918.96$ & 3.44 & - & - \\
\hline pr226 & 37.71 & 30.99 & - & 6.80 & 0.462 & $129,178.20$ & 3.73 & - & 19.42 \\
\hline pr264 & 21.14 & 19.56 & - & - & 0.559 & $62,307.45$ & 3.14 & - & - \\
\hline pr299 & 11.92 & 0.48 & - & 2.09 & 1.093 & $54,854.60$ & 2.60 & - & 62.68 \\
\hline rat195 & 10.36 & 2.98 & 0.38 & 0.51 & 1.462 & $2,599.84$ & 2.70 & 2.63 & 87.23 \\
\hline $\mathrm{rd} 400$ & 11.00 & 16.17 & - & -0.03 & 1.061 & $17,182.49$ & 2.98 & - & 93.66 \\
\hline ts 225 & 0.92 & -0.07 & 1.29 & 14.76 & 1.250 & $128,324.61$ & 2.59 & 2.62 & 912.83 \\
\hline tsp225 & 7.91 & 10.21 & - & -0.36 & 1.271 & $4,216.29$ & 2.83 & - & 39.66 \\
\hline u159 & 0.11 & -0.07 & -0.07 & -0.07 & 1.015 & $42,149.71$ & 2.23 & 2.23 & 33.53 \\
\hline $\begin{array}{l}\text { Time } \\
<=10,000 \\
\mathrm{~s}\end{array}$ & \multicolumn{4}{|c|}{ Time (s) gap best solution (\%) } & \multirow[t]{2}{*}{$\theta:$} & z (length) & \multicolumn{3}{|c|}{ z (angle) } \\
\hline $\begin{array}{l}\text { TSPLIB } \\
\text { case }\end{array}$ & A & B & $\mathrm{C}$ & $\mathrm{D}$ & & A & B & $\mathrm{C}$ & $\mathrm{D}$ \\
\hline \multicolumn{10}{|c|}{$\begin{array}{l}\text { b. At least one run ended in less than } 10,000 \mathrm{~s} \text { and at least one consumed the } \\
\text { limit }\end{array}$} \\
\hline \multirow[t]{2}{*}{ a280 } & 10,000 & 10,000 & 10,000 & 675.0 & 1.319 & $33,523.70$ & 2.46 & 2.46 & 14.41 \\
\hline & 11.98 & 0.08 & 0.00 & 0.00 & & & & & \\
\hline \multirow[t]{2}{*}{ bier127 } & $3,002.3$ & 10,000 & 10,000 & $1,922.6$ & 0.817 & 640.21 & 2.44 & 2.44 & 25.60 \\
\hline & 0.00 & 0.00 & 0.00 & 0.00 & & & & & \\
\hline
\end{tabular}


Table 1 (continued)

\begin{tabular}{|c|c|c|c|c|c|c|c|c|c|}
\hline \multirow{2}{*}{$\begin{array}{l}\text { Time } \\
<=10,000 \\
\mathrm{~s} \\
\text { TSPLIB } \\
\text { case }\end{array}$} & \multicolumn{4}{|c|}{ Time (s) gap best solution (\%) } & \multirow[t]{2}{*}{$\theta:$} & \multirow{2}{*}{$\begin{array}{l}\mathrm{z} \text { (length) } \\
\text { A }\end{array}$} & \multicolumn{3}{|c|}{$\mathrm{z}$ (angle) } \\
\hline & A & $\mathrm{B}$ & $\mathrm{C}$ & $\mathrm{D}$ & & & $\mathrm{B}$ & $\mathrm{C}$ & $\mathrm{D}$ \\
\hline \multirow[t]{2}{*}{ kroA100 } & 10,000 & $1,524.2$ & $1,404.0$ & 122.75 & 1.028 & 544.36 & 2.35 & 2.35 & 20.69 \\
\hline & 23,17 & 0,08 & 0,08 & $\mathbf{0 , 0 8}$ & & & & & \\
\hline \multirow[t]{2}{*}{ rat99 } & 10,000 & 11.5 & 12.2 & 14.03 & 1.455 & 428.87 & 2.56 & 2.56 & 18.29 \\
\hline & -0.62 & -0.62 & -0.62 & -0.62 & & & & & \\
\hline \multicolumn{5}{|c|}{ MIPGap < $0.01 \%$ time $(\mathrm{s})$} & $\theta$ & z (length) & \multicolumn{3}{|c|}{ z (angle) } \\
\hline $\begin{array}{l}\text { TSPLIB } \\
\text { case }\end{array}$ & A & B & $\mathrm{C}$ & $\mathrm{D}$ & & A & B & $\mathrm{C}$ & $\mathrm{D}$ \\
\hline \multicolumn{10}{|c|}{ c. All runs ended in less than $10,000 \mathrm{~s}$} \\
\hline att48 & 503.45 & 344.39 & 409.06 & 123.53 & 1.075 & $33,523.70$ & 2.46 & 2.46 & 14.41 \\
\hline berlin52 & 4.75 & 4.58 & 4.55 & 1.61 & 1.038 & 7544.36 & 2.54 & 2.54 & 10.59 \\
\hline eil101 & 522.25 & 25.56 & 23.95 & 17.63 & 1.500 & 640.21 & 2.44 & 2.44 & 25.60 \\
\hline eil51 & 9.46 & 1.16 & 5.11 & 1.09 & 1.617 & 428.87 & 2.56 & 2.56 & 18.29 \\
\hline eil76 & 3412.25 & 4.64 & 18.37 & 5.44 & 1.487 & 544.36 & 2.35 & 2.35 & 20.69 \\
\hline kroE100 & 460.52 & 2576.74 & 1423.86 & 340.26 & 1.034 & $22,068.75$ & 2.48 & 2.48 & 24.62 \\
\hline pr76 & 633.02 & 793.13 & 851.53 & 637.88 & 1.004 & $108,159.43$ & 2.02 & 2.02 & 14.10 \\
\hline $\operatorname{rd} 100$ & 906.42 & 632.54 & 454.44 & 77.64 & 1.127 & 7910.39 & 2.45 & 2.45 & 30.23 \\
\hline st70 & 269.06 & 520.42 & 348.42 & 136.38 & 1.096 & 677.10 & 2.57 & 2.57 & 17.54 \\
\hline
\end{tabular}

Infeasible runs identified by Gurobi are noted as infeas

Best solution in bold

The gap (\%) column represents the absolute percentage relative deviation of the solution yielded by each model with respect to the optimal TSPLIB tours in terms of distance. Since in general the benchmark tours are not optimal when taking floating distances, gaps become negative in some cases, meaning the solution found is better than the optimal tour with integer distances. Additionally, the table shows the value of the objective function for each formulation: the length of the best solution for the formulation $\mathrm{A}$ and the angle of the respective best solutions in radians for the ECPTSP based formulations B, C, and D. In models (B) and (C), the distance of the objective function to $\pi$ provides an indicator of the improvement with respect to the heuristic taken to calculate $R_{m}{ }^{H}$. Results are complemented with the value of a parameter $\theta$ that characterizes the degree of dispersion of the nodes that define each instance. The details of its calculation and implications will be discussed in Sect. 4.3.

Table 2 presents the characterization of each TSP based on the parameters discussed throughout the paper: the optimal length, optimal mass radius, parameter $\gamma$, upper bound radius used, radius of the convex hull, small radius, and half the maximum distance. 
Table 2 Instance characterization: parameters and coefficients

\begin{tabular}{|c|c|c|c|c|c|c|c|}
\hline TSPLIB & $\mathrm{z}^{*}$ & $R_{m} *$ & $\gamma$ & $\begin{array}{l}\text { Heuristic } \\
R_{m}{ }^{H}\end{array}$ & $\begin{array}{l}\text { Convex } \\
\text { Hull } \\
R_{m} C V\end{array}$ & $\max _{R_{m} S}$ min. & $d \max / 2$ \\
\hline a280 & $2,586.77$ & 411.70 & 1.00389 & 559.82 & 127.48 & 17.89 & 151.17 \\
\hline att 48 & $33,523.70$ & $5,343.43$ & 1.00149 & $6,797.28$ & $4,016.27$ & $1,203.18$ & $4,208.50$ \\
\hline berlin 52 & $7,544.37$ & $1,203.19$ & 1.00206 & $1,486.99$ & 821.11 & 365.00 & 858.02 \\
\hline bier127 & $118,293.52$ & $18,909.40$ & 1.00129 & $22,323.09$ & $9,450.44$ & $6,078.67$ & $9,720.67$ \\
\hline $\operatorname{ch} 130$ & $6,110.86$ & 972.84 & 1.00028 & $1,217.97$ & 428.94 & 75.67 & 469.42 \\
\hline $\operatorname{ch} 150$ & $6,551.41$ & $1,042.83$ & 1.00013 & $1,317.32$ & 395.82 & 86.70 & 424.72 \\
\hline d198 & $15,922.42$ & $2,547.32$ & 1.00520 & $3,553.72$ & $2,169.58$ & $1,138.70$ & $2,130.12$ \\
\hline eil101 & 646.00 & 102.25 & 1.00026 & 130.92 & 42.63 & 12.73 & 45.92 \\
\hline eil51 & 435.12 & 68.49 & 1.00083 & 83.73 & 36.99 & 12.04 & 42.82 \\
\hline eil76 & 546.25 & 86.84 & 1.00047 & 115.80 & 39.72 & 13.45 & 42.64 \\
\hline gil262 & $2,400.62$ & 382.09 & 1.00005 & 527.41 & 124.66 & 19.42 & 133.12 \\
\hline kroA100 & $21,269.22$ & $3,388.91$ & 1.00036 & $4,363.71$ & $1,902.75$ & 361.33 & $2,074.89$ \\
\hline kroA150 & $26,633.80$ & $4,239.50$ & 1.00014 & $5,392.83$ & $1,897.38$ & 302.79 & $2,108.88$ \\
\hline kroA200 & $29,427.98$ & $4,684.03$ & 1.00009 & $5,732.45$ & $1,999.86$ & 300.54 & $2,146.55$ \\
\hline kroB100 & $22,314.76$ & $3,552.72$ & 1.00034 & $4,848.75$ & $1,874.92$ & 461.09 & $2,083.63$ \\
\hline kroB150 & $26,474.37$ & $4,214.22$ & 1.00016 & $5,288.30$ & $1,881.04$ & 357.95 & $2,093.65$ \\
\hline kroB200 & $29,549.01$ & $4,703.28$ & 1.00009 & $5,927.88$ & $1,864.96$ & 253.24 & $2,085.15$ \\
\hline kroC100 & $21,097.48$ & $3,358.91$ & 1.00034 & $4,289.33$ & $1,799.1$ & 357.95 & $2,093.65$ \\
\hline kroD100 & $21,560.95$ & $3,432.74$ & 1.00035 & $4,409.98$ & $1,813.93$ & 397.13 & $2,014.15$ \\
\hline kroE100 & $22,115.35$ & $3,520.98$ & 1.00034 & $4,442.96$ & $1,837.11$ & 458.15 & $2,068.95$ \\
\hline $\operatorname{lin} 105$ & $14,383.00$ & $2,290.58$ & 1.00064 & 3513.01 & $1,404.2$ & 292.50 & $1,594.70$ \\
\hline $\operatorname{lin} 318$ & $42,042.54$ & $6,691.67$ & 1.00006 & $8,741.01$ & $2,206.41$ & 292.50 & $2,432.79$ \\
\hline linhp318 & $42,042.54$ & $6,691.67$ & 1.00006 & $8,741.01$ & $2,206.41$ & 292.50 & $2,432.79$ \\
\hline pr107 & $51,354.37$ & $8,291.72$ & 1.01449 & $8,714.47$ & $5,013.32$ & 282.84 & $5,345.62$ \\
\hline pr124 & $59,468.74$ & $9,473.75$ & 1.00095 & $11,728.08$ & $5,740.17$ & 869.63 & $6,155.80$ \\
\hline pr136 & $96,689.29$ & $15,391.79$ & 1.00021 & $19,193.94$ & $6,367.3$ & $1,093.77$ & $7,038.20$ \\
\hline pr144 & $58,985.22$ & $9,403.34$ & 1.00166 & $10,126.62$ & $5,827.4$ & 300.00 & $6,345.83$ \\
\hline pr152 & $74,229.49$ & $11,850.62$ & 1.00310 & $15,525.87$ & $7,305.25$ & 607.89 & $8,214.65$ \\
\hline pr226 & $80,470.14$ & $12,822.22$ & 1.00117 & $15,637.37$ & $7,818.13$ & $2,300.54$ & $8,711.38$ \\
\hline pr264 & $49,135.00$ & $7,850.53$ & 1.00389 & $9,738.35$ & $4,249.23$ & 651.92 & $4,507.84$ \\
\hline pr299 & $48,313.82$ & $7,689.77$ & 1.00005 & $9,333.28$ & $2,854.67$ & 400.00 & $3,479.40$ \\
\hline pr76 & $108,048.81$ & $17,211.19$ & 1.00085 & $26,695.25$ & $11,302.76$ & $3,905.12$ & $11,337.11$ \\
\hline rat195 & $2,330.55$ & 370.94 & 1.00005 & 443.69 & 128.19 & 14.00 & 152.20 \\
\hline rat99 & $1,226.84$ & 195.30 & 1.00022 & 253.36 & 91.37 & 17.00 & 109.11 \\
\hline $\operatorname{rd} 100$ & 7,991.66 & $1,272.36$ & 1.00035 & $1,611.42$ & 616.63 & 136.06 & 666.84 \\
\hline $\mathrm{rd} 400$ & $15,292.36$ & $2,433.90$ & 1.00002 & $3,059.77$ & 628.98 & 83.12 & 676.52 \\
\hline st70 & 688.48 & 109.64 & 1.00061 & 131.76 & 58.38 & 19.70 & 64.36 \\
\hline ts 225 & $12,7145.93$ & $202,37.47$ & 1.00008 & $24,519.08$ & $7,640.8$ & 500.00 & $8,485.28$ \\
\hline tsp225 & $3,882.85$ & 618.01 & 1.00005 & 763.24 & 217.59 & 28.50 & 259.35 \\
\hline u159 & $42,103.68$ & $6,702.14$ & 1.00017 & $9,429.60$ & $3,082.95$ & 640.31 & $3,342.15$ \\
\hline
\end{tabular}




\subsection{Discussion and influence of the topology of the problem}

In this section, we present a general comparison of the experimentation. Then, we explain a selected parameter to classify the dispersion of nodes, and finally, we use it to group the sets of nodes and plot them to draw some experimentation conclusions.

In Table 3, we compare the results obtained from the forty TSPLIB selected cases for the four formulations. For each formulation, the table shows in how many cases it performs better than each one of the other formulations, alone or tied with another formulation. Formulations (B) and (D) yield better results than (A) in the majority of cases, whereas (C) and (A) are even. Model (D) shows the best performance overall, with 23 cases yielding the best solution, yet the drastic arc elimination strategy leads to infeasibility twice, and in other 4 cases (D) is not able to provide a solution before reaching the time limit. It is therefore a promising approach, but further research concerning the value of is $R_{C}{ }^{S}$ needed. It is worth highlighting that the compact formulation (A) only achieved the best results in 8 out of the 40 cases.

Table 3 Comparison of results between formulations

\begin{tabular}{lllll}
\hline TSPLIB & A & $B$ & C & D \\
\hline A better than & - & 14 & 19 & 10 \\
B better than & 25 & - & 21 & - \\
C better than & 19 & 16 & 22 & 9 \\
D better than & 28 & 8 & 9 & - \\
Total best (including ties) & 8 & & & 23 \\
\hline
\end{tabular}
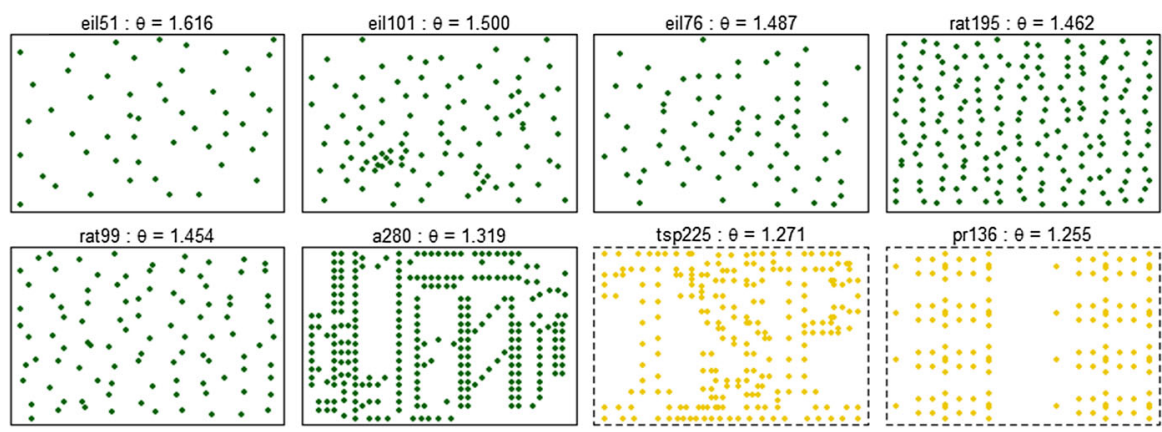

tsp225: $\theta=1.271$

pr136: $\theta=1.255$

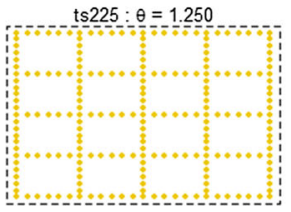

Legend:

- Models B, C \& D

better than Model $A$

Models B, C \& D either better

or worse than Model A
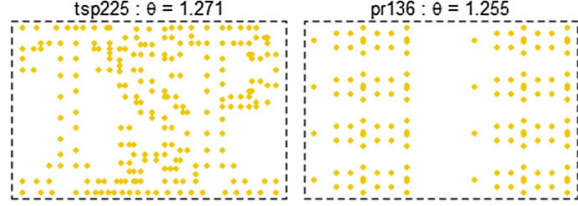

worse than Model A

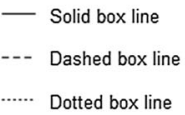

Fig. 6 Sets of nodes from TSPLIB type EUC 2D with less than 400 nodes where $1.2<\theta$ 


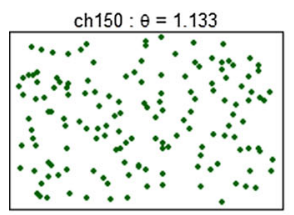

att48: $\theta=1.075$

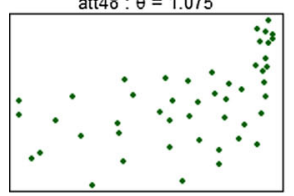

kroA100: $\theta=1.027$
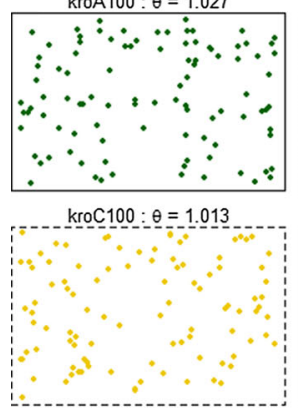

$\operatorname{pr} 76: \theta=1.003$

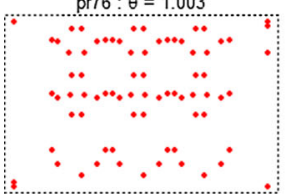

bier127: $\theta=0.817$

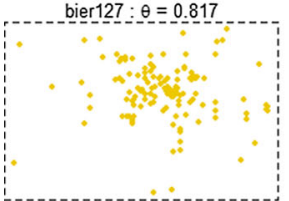

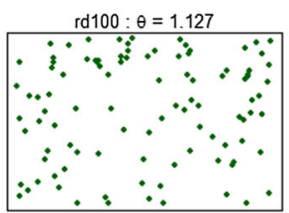

$\operatorname{rd} 400: \theta=1.061$

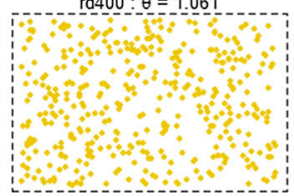

kroB100: $\theta=1.027$

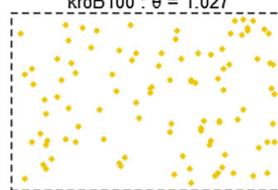

kroB150: $\theta=1.004$

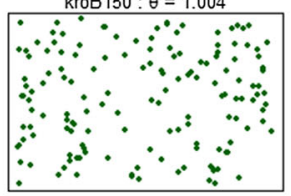

gil262: $\theta=0.994$

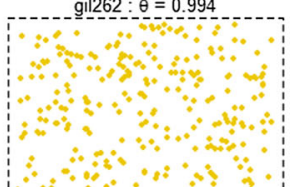

Legend:

Models B, C \& D

better than Model A

Models B, C \& D either better

or worse than Model A

Models B, C \& D

worse than Model A
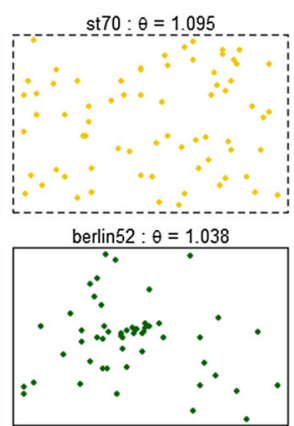

kroA150: $\theta=1.019$

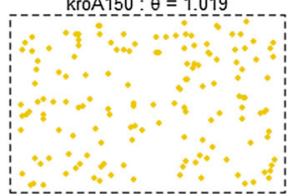

kroB200: $\theta=1.003$

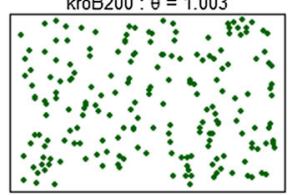

kroA200: $\theta=0.984$
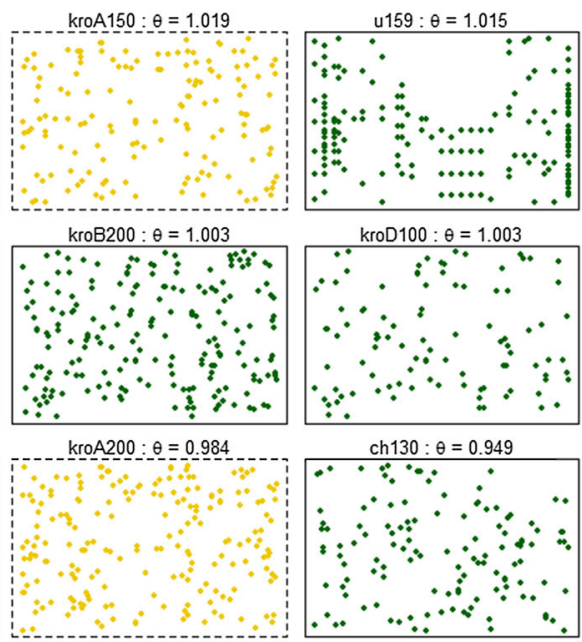

kroD100: $\theta=1.003$

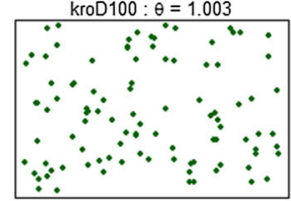

$\operatorname{ch} 130: \theta=0.949$

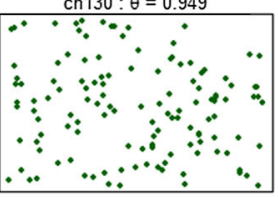

Fig. 7 Sets of nodes from TSPLIB type EUC 2D with less than 400 nodes where $0.8 \leq \theta \leq 1.2$

In conclusion, there does not seem to be a dominant strategy although ECPTSP based formulations show good results in the majority of cases. Nevertheless, these new formulations do not perform well in some of the cases. With the purpose of identifying in which problems the proposed modified formulations have more potential, we analyze the influence of the topology of the problem.

With this aim and following the work of Dry et al. (2012), we calculate a parameter $\theta$ ( $R$ in the original) that characterizes the degree of dispersion of the nodes that define each instance. This parameter leads to a characterization of the set of nodes as clustered, random, or evenly distributed. The parameter adopted is proposed by Clark and Evans (1954) who propose using the distribution of distances from each node to its nearest neighbor as a measure of the spatial dispersion of a set of nodes. To calculate $\theta$, the observed mean nearest neighbor distance $\theta_{o}$ is divided by the mean $\theta_{e}$ 


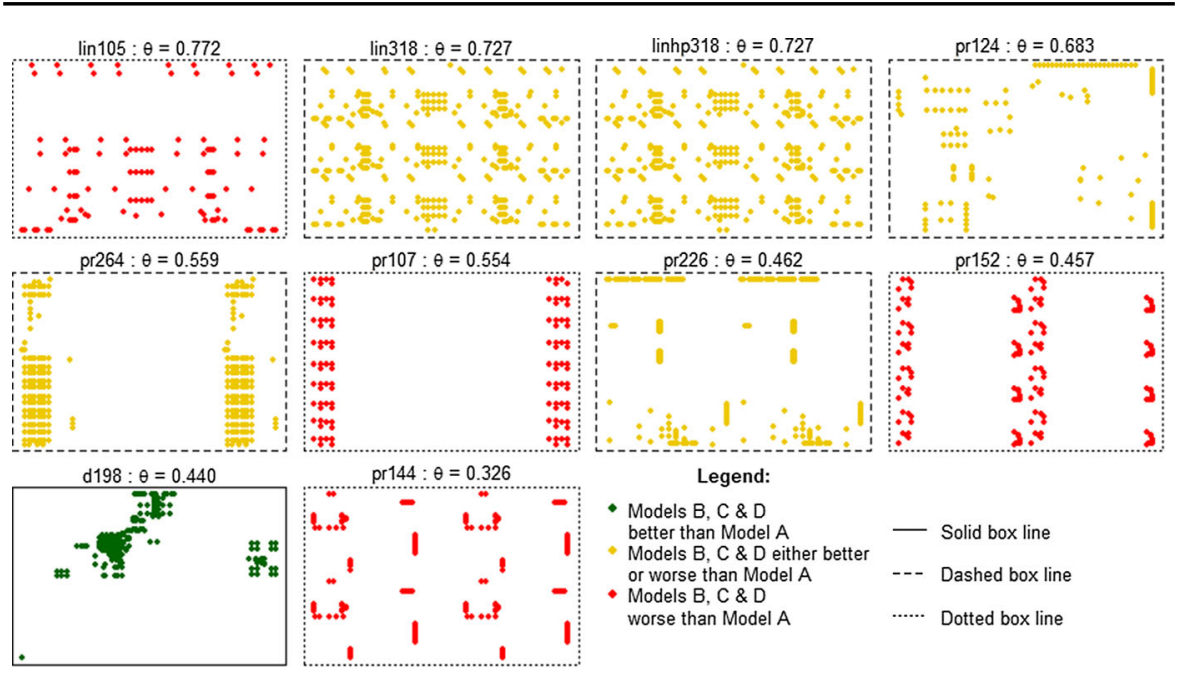

Fig. 8 Sets of nodes from TSPLIB type EUC 2D with less than 400 nodes where $\theta<0.8$

of a Poisson process probability density function that describes the nearest neighbor distance, as expressed in Eq. (19) where $A$ indicates the area.

$$
\vartheta_{o}=\frac{1}{n} \sum_{i \neq j} \min \left\{d_{i j}\right\}, \vartheta_{e}=\frac{1}{2} \sqrt{A / n}, \vartheta=\frac{\vartheta_{o}}{\vartheta_{e}}
$$

The statistic $\theta$ varies between 0 and 2.149, aiming to classify the distribution of the sets of points from highly clustered to highly regular. It represents how long is the average distance to the nearest neighbor compared to a random set of nodes. The closer to 1 is $\theta$ the more randomly are the nodes distributed; if $\theta$ is close to 0 , the nodes are highly clustered; if $\theta$ is high it means the average distance to the nearest neighbor is proportionally high, and then the nodes are evenly distributed.

We classify the 40 TSPLIB instances according to their $\theta$ value and, similarly to Dry et al. (2012) we define three groups, respectively, $\{\theta<0.8\},\{0.8 \leq \theta \leq 1.2\}$, and $\{1.2<\theta\}$. Figures 3,4 , and 5 show the plots of the three groups ordered by their $\theta$ value. We use a graphic code to signify the performance of the ECPTSP formulations: if formulations (B, C, and D) obtain better results than formulation (A) in terms of gap solution or computing time when they provide the same gap solution, the plot rectangle edges are continuous and dots are dark green colored; dashed edges and orange dots signify that at least one ECPTSP formulation (B, C and D) obtain better results than formulation (A) or all four models have identical results, and dotted edges and red color is used when formulation (A) performs the best.

Figure 6 shows that for high values of $\theta$, ECPTSP formulations (B, C or D) yield satisfactory results. In six of the nine instances the formulation (A) performs the worst. In the results of Table 1 we see how in cases eil51, eil101, eil76, rat195, and rat99, either gaps or computing times decrease substantially with these formulations. 
In Fig. 7, there are ten sets in continuous edge (green), ten sets in dashed (orange), and only one set in dotted (red). Regarding this last case, pr76, we observe in Table 1 a similar computation time for all formulations, although (A) performs slightly better than (B, C, and D). Sets st70, rd400, kroA150, gil262, and kroA200 behave the best when using model (D), and ch130 solution improvement for models (B) and (C) is significative.

Figure 8 reveals that high clusterization has a negative impact on the performance of models (B, C, and D). The distribution of the nodes presents peculiar patterns. Only in one of the ten instances, the three ECPTSP formulations show better behavior than (A) and in four of the cases (A) yields the best results. Model (D) may be infeasible by using the selected $R_{c}{ }^{S}$. On the other hand, if $\theta>1$, model (D) performs better than model (A) in all cases except in ts 225 , where a regular pattern is identified, and in pr76, where the performance of all models results in similar.

\section{Conclusions}

Through a geometric transformation based on polygonal linkages, we can inscribe each transformed TSP tour as an open polygonal chain in a circle of a given radius (Equivalent Inscribed Polygonal Chain, EIPC) as well as find its Equivalent Cyclic Polygon (ECP). This transformation leads to a modified formulation for the extensively studied classic Euclidean TSP in which the objective function is expressed as a summation of angles instead of distances.

The modified formulation leads in turn to the definition of a new family of heuristic models (ECPTSP formulations). Experimentation with forty cases of TSPLIB shows that using the ECPTSP formulations may help to obtain better or optimal solutions faster than compact formulations. Using ECP properties mixed with a drastic link elimination shows promising results in some cases. We also verify that a parameter based on the distribution of the nearest neighbor distances is of practical interest in TSP topology characterization. Particularly, it shows that the formulations based on the proposed tour geometric transformation perform better when the nodes are randomly or evenly distributed, whereas it tends to yield worse results in highly clustered problems.

The performance of the heuristic formulation reveals the interest of exploring ways to set the small radius parameter so that it improves the performance of the solving procedure while not incurring in infeasibility or leading to high computation times to provide a first feasible solution. The definition of the ECP opens the way for further research on TSP, exploring new properties and new heuristics. The number of formulations, and TSP extensions such as the Vehicle Routing Problem (VRP), also bring the interest of investigating the applicability of the ECP transformation to achieve efficient results in other models and variants.

Acknowledgements Grant RTI2018-094614-B-I00 (SMASHING) into the "Programa Estatal de I+D+i Orientada a los Retos de la Sociedad" funded by MCIN/AEI/ 10.13039/501100011033.

Funding Open Access funding provided thanks to the CRUE-CSIC agreement with Springer Nature. 
Open Access This article is licensed under a Creative Commons Attribution 4.0 International License, which permits use, sharing, adaptation, distribution and reproduction in any medium or format, as long as you give appropriate credit to the original author(s) and the source, provide a link to the Creative Commons licence, and indicate if changes were made. The images or other third party material in this article are included in the article's Creative Commons licence, unless indicated otherwise in a credit line to the material. If material is not included in the article's Creative Commons licence and your intended use is not permitted by statutory regulation or exceeds the permitted use, you will need to obtain permission directly from the copyright holder. To view a copy of this licence, visit http://creativecommons.org/licenses/ by/4.0/.

\section{References}

Anbuudayasankar SP, Ganesh K, Mohapatra S (2014) Models for practical routing problems in logistics: Design and practices. Model Pract Routing Probl Logist Des Pract 9783319050355:1-165. https:// doi.org/10.1007/978-3-319-05035-5

Applegate DL, Bixby RE, Chvátal V, Cook WJ (2006) The Traveling Salesman Problem: A Computational Study. Princeton Unversity, Princeton, NJ

Asani EO, Okeyinka AE, Adebiyi AA (2020) A Construction tour technique for solving the travelling salesman problem based on convex hull and nearest neighbour heuristics. Int Conf Math Comput Eng Comput Sci ICMCECS. https://doi.org/10.1109/ICMCECS47690.2020.240847

Biggs NL, LLoyd EK, Wilson RJ (1976) Graph Theory 1736-1936. Clarendon Press, Oxford

Clark PJ, Evans FC (1954) Distance to nearest neighbor as a measure of spatial relationships in populations. Ecology 35:445-453. https://doi.org/10.2307/1937660

Dantzig GB, Fulkerson DR, Johnson SM (1954) Solution of a large-scale traveling-salesman problem. Oper Res 2:393-410. https://doi.org/10.1287/opre.2.4.393

Desrochers M, Laporte G (1991) Improvements and extensions to the Miller-Tucker-Zemlin subtour elimination constraints. Op Res Lett 10(1):27-36. https://doi.org/10.1016/0167-6377(91)90083-2

Dry M, Preiss K, Wagemans J (2012) Clustering, randomness, and regularity: spatial distributions and human performance on the traveling salesperson problem and minimum spanning tree problem. J Problem Solving 4:1-17. https://doi.org/10.7771/1932-6246.1117

Dubois-Lacoste J, Hoos HH, Stützle T (2015) On the empirical scaling behaviour of state-of-the-art local search algorithms for the euclidean TSP. GECCO 2015 - Proc 2015 Genet Evol Comput Conf 377-384. https://doi.org/10.1145/2739480.2754747

Edelsbrunner H (1987) Algorithms in Combinatorial Geometry. Springer, Berlin Heidelberg

Edelsbrunner H (2014) A Short Course in Computational Geometry and Topology. Springer International Publishing, Cham

Fox KR, Gavish B, Graves SC (1980) An $n$-constraint formulation of the (time-dependent) traveling salesman problem. Oper Res 28(4):1018-1021. https://doi.org/10.1287/opre.28.4.1018

Gavish B, Graves SC (1978) The travelling salesman problem and related problems. Operations Research Center Working Paper OR 078-78. http://hdl.handle.net/1721.1/5363

Gouveia L, Pires JM (2001) The asymmetric travelling salesman problem: on generalizations of disaggregated Miller-Tucker-Zemlin constraints. Discret Appl Math 112(1-3):129-145. https://doi.org/10. 1016/S0166-218X(00)00313-9

Gurobi Op (2021a) Gurobi optimizer reference Manual. www.gurobi.com. Accessed 30 Jul 2021

Gurobi Op (2021b) MIPGap. https://www.gurobi.com/documentation/9.1/refman/mipgap2.html. Accessed 30 Jul 2021

Hart WE, Carl DL, Watson JP, Woodruff DL, Hackebeil GA, Nicholson BL, Siirola JD (2017) Pyomo Optimization modeling in python. Second Edition. Vol. 67. Springer, 2017.

Junger M, Reinelt G, Rinaldi G (1995) The traveling salesman problem. In: Ball MO, Magnanti TL, Monma CL, Nemhauser GL (eds) Network models handbooks in operations research and management science, vol 7. North-Holland, Amsterdam, pp 225-330

Langevin A, Soumis F, Desrosiers J (1990) Classification of travelling salesman problem formulations. Oper Res Lett 9:127-132

Laporte G (1992) The traveling salesman problem: An overview of exact and approximate algorithms. Eur J Oper Res 59(2):231-247. https://doi.org/10.1016/0377-2217(92)90138-Y 
Lawler EL, Lenstra JK, Rinnooy Kan AHG, Shmoys DB (eds) (1991) The traveling salesman problem. A guided tour of combinatorial optimization. John Wiley and Sons, Chichester

Lenstra JK, Kan AHGR (1975) Some simple applications of the travelling salesman problem. J Oper Res Soc 26(4):717-733

Levcopoulos C, Lingas A, Mitchell JSB (2002) Adaptive algorithms for constructing convex hulls and triangulations of polygonal chains. Lect Notes Comput Sci 2368:80-89. https://doi.org/10.1007/3540-45471-3_9

Macnab DS (1981) Cyclic polygons and related questions. Math Gaz 65:22-28. https://doi.org/10.2307/ 3617929

Miller CE, Tucker AW, Zemlin RA (1960) Integer programming formulations and traveling salesman problems. J Assoc Comput Mach 7:326-329. https://doi.org/10.1145/321043.321046

Norback JP, Love RF (1977) Geometric approaches to solving the traveling salesman problem. Manage Sci 23(11):1208-1223. https://doi.org/10.1287/mnsc.23.11.1208

Öncan T, Altınel İK, Laporte G (2009) A comparative analysis of several asymmetric traveling salesman problem formulations. Comput Oper Res 36(3):637-654. https://doi.org/10.1016/j.cor.2007.11.008

Orman AJ, Williams HP (2007) A Survey of Different Integer Programming Formulations of the Travelling Salesman Problem. Optim Econ Financ Anal Adv Comput Manage Sci. https://doi.org/10.1007/3540-36626-1_5

Padberg M, Sung TY (1991) An analytical comparison of different formulations of the travelling salesman problem. Math Program 521(52):315-357. https://doi.org/10.1007/BF01582894

Panina GY, Khimshiashvili GN (2012) On the area of a polygonal linkage. Dokl Math 85:120-121. https:// doi.org/10.1134/S1064562412010401

Papadimitriou CH (1977) The Euclidean travelling salesman problem is NP-complete. Theor Comput Sci 4:237-244. https://doi.org/10.1016/0304-3975(77)90012-3

Pinelis I (2005) Cyclic polygons with given edge lengths: Existence and uniqueness. J Geom 821(82):156-171. https://doi.org/10.1007/S00022-005-1752-8

R Core Team (2020). R: A language and environment for statistical computing. R Foundation for Statistical Computing, Vienna, Austria.URL https://www.R-project.org/.

Raman V, Gill NS (2017) Review of different heuristic algorithms for solving Travelling Salesman Problem. Int J Adv Res Comput Sci 8:423-425

Reinelt G (1991) TSPLIB - A traveling salesman problem library. ORSA J Comp 3:376-384. https://doi. org/10.1287/ijoc.3.4.376

Reinelt G (1994) The traveling salesman: Computational solutions for TSP applications. Springer, Berlin Heidelberg

Rosenkrantz DJ, Stearns RE, Lewis PM II (1977) An analysis of several heuristics for the traveling salesman problem. SIAM J Comput 6:563-581. https://doi.org/10.1137/0206041

Publisher's Note Springer Nature remains neutral with regard to jurisdictional claims in published maps and institutional affiliations. 